T. Kurogi

Nagoya Math. J.

Vol. 81 (1981), 27-43

\title{
ON SOME TYPES OF GEODESICS ON RIEMANNIAN MANIFOLDS
}

\author{
TETSUNORI KUROGI
}

\section{§ 0. Introduction}

For a given Riemannian manifold $M$ and its submanifold $N$, one can find various types of geodesics on $M$ starting from any point of $N$ and ending in any point of $N$. For example, geodesics which start perpendicularly from $N$ and end perpendicularly in $N$ are treated by many mathematicians. K. Grove has stated a condition in a general case for the existence of such a geodesic ([4]), where he has used the method of the infinite dimensional critical point theory. This method is very useful for the study of geodesics and many geometricians have used it successfully. It has two aspects: one is an existence theory and the other is a quantitative theory, which one can find, for instance, in the excellent theory for closed geodesics of W. Klingenberg ([1], [7]) and so on.

On the other hand the works of K. Grove ([2], [4]) suggest us that this method is applicable to qualitative questions for geodesics. Here we shall study some types of geodesics from this point of view.

Let $M$ be a complete Riemannian manifold and let $N$ be a closed submanifold, then it seems very interesting to ask "Do there exist geodesics on $M$ which start from $N$ with one angle and end in $N$ with the same angle?"

Unfortunately for our problem, we cannot use the infinite dimensional method directly, because it is difficult to find a satisfactory infinite dimensional manifold. Under some nice conditions, however, we can apply this method to our problem: that is to find a good isometry on $M$ with respect to $N$.

We don't know whether our idea is extensible to a more general theory or not, but we are sure to give a little clue to solve a more general problem. And at the same time our work makes an example of the in-

Received September 13, 1977. 
finite dimensional method to be useful for a qualitative study of geodesics.

This paper is divided into four sections. In the first section, we point out an infinite dimensional submanifold of $L_{1}^{2}(I, M)$, which is a Hilbert manifold of absolutely continuous maps from the unit interval $I=[0,1]$ to $M$ with square integrable derivative, and we point out its related geodesic on $M$, which is obtained as a critical point for the energy function on this infinite dimensional manifold. We call it "geodesic with the same angle" (See Definition 1-1). In the next section, we discuss the existence of such a geodesic in the same way as K. Grove ([2]). We get our main theorem (Theorem A) and some corollaries. In the third section, we treat of geodesic loops (self-intersecting geodesics) as a special case of the second section (Theorem B). And in the last section, we consider very special geodesics which we call $N$-I-geodesics (normal connecting invariant geodesics) and give a condition for the existence of $N$-I-geodesics (Theorem $\mathrm{C}$ ).

Our main results are contained in

Theorem A. Let $M$ be a complete Riemannian manifold, $N$ a connected compact closed submanifold of codimension 1 with $\partial N=\phi$ and $f$ an isometry on $M$ with $f(N)=N$. If $\pi_{1}(M)=\{0\}$, then there exists a non-trivial geodesic with the same angle for $N$ when $f$ has finite fixed points on $N$ except that it has exactly one fixed point.

Theorem B. Let $M$ be a complete Riemannian manifold and let $N$ be a connected compact closed submanifold of codimension 1 with $\partial N=\phi$. If $\pi_{k}(M) \neq 0$ for some $k \geqq 1$, then there exists a non-trivial geodesic loop starting from $N$ with the same angle for $N$.

Let $\Gamma(M)$ be a group of isometries of $M$ and let $\Gamma_{N}(M)$ be a subgroup of $\Gamma(M)$ such that any element of $\Gamma_{N}(M)$ leaves $N$ invariant.

Theorem C. Let $M$ be a complete Riemannian manifold and let $N$ be a connected compact closed submanifold of codimension 1 with $\partial N=\phi$. If $\Gamma_{N}(M)$ acts on $N$ transitively and $N$ is not the same homotopy type as $M$, then there exists an element $f$ of $\Gamma_{N}(M)$ such that it has a N-I-geodesic.

\section{§1. Hilbert submanifold and energy function}

Let $M$ be a complete Riemannian manifold with a Riemannian metric $\langle$,$\rangle and let L_{1}^{2}(I, M)$ be the set of absolutely continuous maps from the unit interval $I=[0,1]$ to $M$ with square integrable derivative. Then it is 
known that $L_{1}^{2}(I, M)$ is a complete Hilbert Riemannian manifold with the Riemannian structure given by

$$
\ll X_{\alpha}, Y_{\alpha} \gg=\int_{0}^{1}\left\{\left\langle X_{\alpha}(t), Y_{\alpha}(t)\right\rangle+\left\langle\nabla_{\alpha} X_{\alpha}(t), \nabla_{\alpha} Y_{\alpha}(t)\right\rangle\right\} d t
$$

where $X_{\alpha}, Y_{\alpha}$ are elements of the tangent space $T_{\alpha} L_{1}^{2}(I, M)$ at $\alpha \in L_{1}^{2}(I, M)$, which is a linear space of absolutely continuous vector fields along $\alpha$ on $M$ with square integrable covariant derivative $\nabla_{\alpha} X_{\alpha}$.

Since the map $P: L_{1}^{2}(I, M) \rightarrow M \times M$ defined by $P(\alpha)=(\alpha(0), \alpha(1))$ for all $\alpha \in L_{1}^{2}(I, M)$ induces a surjective linear map $P_{* \alpha}: T_{\alpha} L_{1}^{2}(I, M) \rightarrow T_{\alpha(0)} M \times$ $T_{\alpha(1)} M$ which has a splitting kernel, $P$ is a submersion so that the preimage of any submanifold of $M \times M$ is a submanifold of $L_{1}^{2}(I, M)$.

Let $N$ be a closed submanifold of $M$ with $\partial N=\phi$ and let $f$ be an isometry on $M$ with $f(N)=N$. We introduce the following sets written as $\Lambda(M, f)$ and $\Lambda_{N}(M, f)$ respectively:

$$
\begin{aligned}
\Lambda(M, f) & =\left\{\alpha \in L_{1}^{2}(I, M) \mid f(\alpha(0))=\alpha(1)\right\} \\
\Lambda_{N}(M, f) & =\{\alpha \in \Lambda(M, f) \mid \alpha(0) \in N\} .
\end{aligned}
$$

Let $G(M, f)$ and $G(M, N, f)$ be graphs $\{(x, f(x)) \mid x \in M\}$ and $\{(x, f(x)) \mid x \in N\}$ of an isometry $f$ respectively, then $\Lambda(M, f)$ and $\Lambda_{N}(M, f)$ are submanifolds of $L_{1}^{2}(I, M)$ because $P^{-1}(G(M, f))=\Lambda(M, f)$ and $P^{-1}(G(M, N, f))=\Lambda_{N}(M, f)$. The space $\Lambda(M, f)$ is originally introduced by $\mathrm{K}$. Grove for the study of invariant geodesics. Here we consider the space $\Lambda_{N}(M, f)$ for our problem. Then we have

Proposition 1-1. $\Lambda_{N}(M, f)$ is a Hilbert Riemannian submanifold of $L_{1}^{2}(I, M)$ and its tangent space $T_{\alpha} \Lambda_{N}(M, f)$ at $\alpha$ is given by

$$
T_{\alpha} \Lambda_{N}(M, f)=\left\{X_{\alpha} \in T_{\alpha} L_{1}^{2}(I, M) \mid\left(X_{\alpha}(0), X_{\alpha}(1)\right) \in T_{\alpha(0)} N \times T_{\alpha(1)} N, f_{*} X_{\alpha}(0)=X_{\alpha}(1)\right\} \bullet
$$

Define an energy function $E: L_{1}^{2}(I, M) \rightarrow R$ by $E(\alpha)=1 / 2 \int_{0}^{1}\|\dot{\alpha}(t)\|^{2} d t$, then it is a $C^{\infty}$-map. In particular we consider the restriction of $E$ on $\Lambda_{N}(M, f)$ and we also write it $E$. It is known that

$$
d E_{\alpha}\left(X_{\alpha}\right)=\int_{0}^{1}\left\langle\nabla_{\alpha} X_{\alpha}(t), \dot{\alpha}(t)\right\rangle d t
$$

for all $X_{\alpha} \in T_{\alpha} L_{1}^{2}(I, M)$.

Then the following regularity is given by $\mathrm{K}$. Grove ([2]). 
Proposition 1-2 (regularity). If $V$ is a submanifold of $L_{1}^{2}(I, M)$ such that $T_{\alpha} V$ for $\alpha \in V$ contains all $X_{\alpha} \in T_{\alpha} L_{1}^{2}(I, M)$ with $X_{\alpha}(0)=0$ and $X_{\alpha}(1)=$ 0 , and if $\alpha$ is a critical point for $\left.E\right|_{V}$, then $\alpha$ is $C^{\infty}$ and $\alpha$ is a geodesic.

Now we define a new type of geodesic in order to see what sort of geodesics is a critical point of $E$ on $\Lambda_{N}(M, f)$. Let $M$ be a complete Riemannian manifold and let $N$ be a closed submanifold of codimension 1 . At a point $x \in N$, we denote by $e_{x}$ a unit normal to $T_{x} N$ in $T_{x} M$.

Definition 1-1. A geodesic $\alpha: I \rightarrow M$ with $(\alpha(0), \alpha(1)) \in N \times N$ is called "geodesic with the same angle for $N$ " (simply "geodesic with the same angle"), if

$$
\left\langle\dot{\alpha}(0), \boldsymbol{e}_{\alpha(0)}\right\rangle+\left\langle\dot{\alpha}(1), \boldsymbol{e}_{\alpha(1)}\right\rangle=0
$$

or

$$
\left\langle\dot{\alpha}(0), \boldsymbol{e}_{\alpha(0)}\right\rangle-\left\langle\dot{\alpha}(1), \boldsymbol{e}_{\alpha(1)}\right\rangle=0 .
$$

When $N$ is a totally geodesic submanifold, any geodesic on $N$ is always a geodesic with the same angle.

Combining Propositions 1-1 and 1-2, we get

TheOREM 1-3. Let $M$ be a complete Riemannian manifold, $N$ a closed submanifold of codimension 1 and $f$ an isometry of $M$ with $f(N)=N$. If $\alpha \in \Lambda_{N}(M, f)$ is a critical point for $E: \Lambda_{N}(M, f) \rightarrow R$, then $\alpha$ is a geodesic with the same angle.

Proof. Assume that $\alpha \in \Lambda_{N}(M, f)$ is a critical point for $E$. By Proposition $1-2, \alpha$ is a geodesic on $M$ so that for any tangent vector $X_{\alpha} \in$ $T_{\alpha} \Lambda_{N}(M, f)$,

$$
\begin{aligned}
d E_{\alpha}\left(X_{\alpha}\right) & =\int_{0}^{1}\left\langle\nabla_{\alpha} X_{\alpha}(t), \dot{\alpha}(t)\right\rangle d t \\
& \left.=\int_{0}^{1}\left\{\nabla_{\alpha} X_{\alpha}(t), \dot{\alpha}(t)\right\rangle+\left\langle X_{\alpha}(t), \nabla \dot{\alpha}(t)\right\rangle\right\} d t \\
& =\int_{0}^{1} \frac{d}{d t}\left\langle X_{\alpha}(t), \dot{\alpha}(t)\right\rangle d t \\
& =\left\langle X_{\alpha}(1), \dot{\alpha}(1)\right\rangle-\left\langle X_{\alpha}(0), \dot{\alpha}(0)\right\rangle \\
& =\left\langle X_{\alpha}(1), \dot{\alpha}(1)-f_{*} \dot{\alpha}(0)\right\rangle .
\end{aligned}
$$

Since $\alpha$ is a critical point, we have $\dot{\alpha}(1)-f_{*} \dot{\alpha}(0) \perp T_{\alpha(1)} N$ from (2). If $e$ 
is unit normal to $T_{\alpha(0)} N$, then $f_{*} e$ is unit normal to $T_{\alpha(1)} N$ and so there exists a number $\lambda$ such that $\dot{\alpha}(1)-f_{*} \dot{\alpha}(0)=\lambda f_{*} e$. From this, we have $\|\dot{\alpha}(1)\|^{2}$ $=\|\dot{\alpha}(0)\|^{2}+2 \lambda\langle\dot{\alpha}(0), e\rangle+\lambda^{2}$. Since $\alpha$ is a geodesic, $\|\dot{\alpha}(0)\|=\|\dot{\alpha}(1)\|$ and hence we have $\lambda=0$ or $\lambda=-2\langle\dot{\alpha}(0), e\rangle$. If $\lambda=0$, then we have $\dot{\alpha}(1)=f_{*} \dot{\alpha}(0)$ which implies that the geodesic $\alpha$ is invariant under $f$ and it is also a geodesic with the same angle. On the other hand, since

$$
\begin{aligned}
\lambda & =\left\langle\lambda f_{*} \boldsymbol{e}, f_{*} \boldsymbol{e}\right\rangle \\
& =\left\langle\dot{\alpha}(1)-f_{*} \dot{\alpha}(0), f_{*} \boldsymbol{e}\right\rangle \\
& =\left\langle\dot{\alpha}(1), f_{*} \boldsymbol{e}\right\rangle-\langle\dot{\alpha}(0), \boldsymbol{e}\rangle,
\end{aligned}
$$

we have $\left\langle\dot{\alpha}(1), f_{*} \boldsymbol{e}\right\rangle+\langle\dot{\alpha}(0), \boldsymbol{e}\rangle=0$ if $\lambda=-2\langle\dot{\alpha}(0), \boldsymbol{e}\rangle$. Therefore $\alpha$ is a geodesic with the same angle.

[Q.E.D.]

Remark. As seen in the proof of Theorem 1-3, a critical point $\alpha \epsilon$ $\Lambda_{N}(M, f)$ for $E$ is an invariant geodesic under $f$ when $\lambda=0$. And here the concept of "geodesic with the same angle" contains partly the concept of "invariant geodesic".

When $f=$ identity on $M$ in Theorem $1-3, \alpha$ is a self-intersecting geodesic with the same angle at a point of $N$ if $\alpha \in \Lambda_{N}(M, f)$ is a critical point for $E$. (On this we shall study further in $\S 3$.) In a special case a closed geodesic on $M$ passing through $N$ may occur as a critical point of $\Lambda_{N}(M, f)$ for $E$.

Furthermore we have the following interesting results.

Corollary 1-4. Let $M, N$ and $f$ be the same as Theorem 1-3. If $\alpha$ is a critical point with $\dot{\alpha}(0) \in T_{\alpha(0)} N$, then $\alpha$ is an f-invariant geodesic on $M$ such that both ends are tangent to $N$.

Proof. If $\dot{\alpha}(0)$ is tangent to $N, \lambda$ must be 0 so that $\alpha$ is an $f$-invariant geodesic. On the other hand, since $\lambda=\left\langle\dot{\alpha}(1), f_{*} e\right\rangle-\langle\dot{\alpha}(0), e\rangle$, we get $\left\langle\dot{\alpha}(1), f_{*} e\right\rangle=0$ if $\dot{\alpha}(0) \in T_{\alpha(0)} N$, and so $\dot{\alpha}(1)$ is tangent to $N$.

[Q.E.D.]

From Remark and Corollary 1-4 we have

Corollary 1-5. Let $M$ and $N$ be the same as Theorem 1-3. If $\alpha \in$ $\Lambda_{N}(M, \mathrm{id})$ is a critical point for $E$ starting tangent to $N$, then $\alpha$ is a closed geodesic on $M$ passing through $N$.

Proof. Note that an $f$-invariant geodesic is a closed geodesic when $f=\mathrm{id}$. Then our conclusion follows from Remark and Corollary 1-4.

[Q.E.D.] 


\section{$\S 2$. Geodesic with the same angle}

Let $M$ be a complete Riemannian manifold and let $N$ be a closed submanifold of $M$, then we propose a question: "Do there exist geodesics on $M$ which start from $N$ with one angle and end in $N$ with the same angle?". Here we construct an existence theory for the above problem under some conditions by using an isometry on $M$. For that in an analogous way to Grove's method ([2]) we study the space $\Lambda_{N}(M, f)$ and its energy function as we have seen in $\S 1$ : that is the critical point theory for $\left(\Lambda_{N}(M, f), E\right)$. In this theory it is very important to find the conditions to guarantee the existence of critical point for $E$. In general, such a fundamental analytic condition is known as Palais-Smale's condition (usually called "Condition (C)"). Condition (C) is that whenever $\left\{\alpha_{n}\right\}$ is a sequence on which $E$ is bounded and for which $\left\|\left|\operatorname{grad} E_{\alpha_{n}}\right|\right\|_{\alpha_{n}} \rightarrow 0$ when $n \rightarrow \infty,\left\{\alpha_{n}\right\}$ has a convergent subsequence, where $\|\cdot \cdot\| \|_{\alpha_{n}}$ denotes the norm in $T_{\alpha} \Lambda_{N}(M, f)$. Then by Theorem 2-4 ([2]), we have

Proposition 2-1. The energy function $E: \Lambda_{N}(M, f) \rightarrow \boldsymbol{R}$ satisfies the Condition (C) if $N$ is compact.

Now we see what is the $E^{-1}(0)$ for $E: \Lambda_{N}(M, f) \rightarrow R$. If $\alpha$ is an element of $\Lambda_{N}(M, f)$ with $E(\alpha)=0, \alpha$ must be a constant map which we identify with a point of $N$ and $f(\alpha(0))=\alpha(1)$ and so we have that $p=\alpha(t)$ for any $t \in I$ is a fixed point of $f$ in $N$. Conversely if $p$ is a fixed point of $f$ in $N$, then we can define a map $\alpha \in \Lambda_{N}(M, f)$ by $\alpha(t)=p$ for any $t \in I$ so that $\alpha$ is an element of $E^{-1}(0)$. Thus we can identify $E^{-1}(0)$ with the set of fixed points for $f$ in $N$. We denote the set of fixed points for $f$ in $N$ by $F(N, f)$. (The $F(N, f)$ will be $F(f) \cap N$ where $F(f)$ is the set of fixed points for $f$.) Let $F_{i}(N, f)$ be a connected component of $F(N, f)$, then $F(N, f)=\sum F_{i}(N, f)$. And it is clear that $F_{i}(N, f)$ is a totally geodesic submanifold of $N$ by using a relation $\exp _{p} \circ f_{*}=f \circ \exp _{p}$ when $f(p)=p \in N$ where $\exp _{p}$ is an exponential map with respect to the induced metric on $N$ from $M$. Thus we have that each $F_{i}(N, f)$ is a critical submanifold of $\Lambda_{N}(M, f)$.

Next we see the index of $F_{i}(N, f)$ and the non-degeneracy for $F_{i}(N, f)$. For that we must calculate the Hessian $H(E)_{\alpha}$ for $E$ at a critical point $\alpha \in \Lambda_{N}(M, f)$. Calculate $H(E)_{\alpha}\left(X_{\alpha}, Y_{\alpha}\right)=\left(\partial^{2} / \partial t \partial s\right) E(\omega(t, s))_{(t, s)=(0,0)}$ by using a 2-parameter variation $\omega: I \times I \rightarrow \Lambda_{N}(M, f)$ with $\omega(0,0)=\alpha, \omega_{t}(0,0)=X_{\alpha}$, $\omega_{s}(0,0)=Y_{\alpha}$, then we have 
(3)

$$
\begin{aligned}
H(E)_{\alpha}\left(X_{\alpha}, Y_{\alpha}\right) & =\int_{0}^{1}\left\langle\nabla_{\alpha} X_{\alpha}(t), \nabla_{\alpha} Y_{\alpha}(t)\right\rangle d t \\
& -\int_{0}^{1}\left\langle R\left(X_{\alpha}(t), \dot{\alpha}(t)\right) \dot{\alpha}(t), Y_{\alpha}(t)\right\rangle d t
\end{aligned}
$$

for any tangent vectors $X_{\alpha}, Y_{\alpha} \in T_{\alpha} \Lambda_{N}(M, f)$ if $\alpha \in \Lambda_{N}(M, f)$ is a critical point for $E$, where $R($, ) is a Riemannian curvature for $M$. This form is already obtained by many people (see [1], [2]). Moreover as to the Hessian for the energy function we have a decomposition lemma.

LEMma 2-2. Let $X$ be a submanifold of $L_{1}^{2}(I, M)$ and $E: X \rightarrow R$ be an energy function. If the Hessian $H(E)_{x}(v, w)$ for $E$ is given by $\int_{0}^{1}\langle\nabla v(t)$, $\nabla w(t)\rangle d t$ at $x \in X$ for any vectors $v, w \in T_{x} X$, then there exists a compact operator $k_{x}: T_{x} X \rightarrow T_{x} X$ such that $h(E)_{x}=1+k_{x}$ where $h(E)_{x}$ is defined by $\left\langle h(E)_{x} v, w \gg=H(E)_{x}(v, w)\right.$. Here $\ll, \gg$ is the induced metric on $X$ by $L_{1}^{2}(I, M)$.

Proof. Since $\left\langle v, w \gg=\int_{0}^{1}\{\langle v(t), w(t)\rangle+\langle\nabla v(t), \nabla w(t)\rangle\} d t\right.$ for any vectors $v, w \in T_{x} X$, we have $\ll\left(h(E)_{x}-1\right) v, w \gg=-\int_{0}^{1}\langle v(t), w(t)\rangle d t$.

On the other hand we define a continuous bilinear form on $T_{x} X$ by $(v, w) \rightarrow-\int_{0}^{1}\langle v(t), w(t)\rangle d t$, then there exists a continuous linear map $k_{x}$ : $T_{x} X \rightarrow T_{x} X$ such that $\left\langle k_{x} v, w \gg=-\int_{0}^{1}\langle v(t), w(t)\rangle d t\right.$. Thus we have $h(E)_{x}$ $=1+k_{x}$.

Next we see that $k_{x}$ is a compact operator. Let $\ll, \gg_{0}$ be the inner product on $T_{x} X$ defined by $\langle v, w\rangle_{0}=\int_{0}^{1}\langle v(t), w(t)\rangle d t$, then note that the identity map from $T_{x} X$ with $\ll, \gg$ to $T_{x} X$ with $\ll, \gg_{0}$ is a compact operator. Thus we have

$$
\left\|\left|k_{x} v\right|\right\| \leqq \text { const } \cdot\|v \mid\|_{0} \quad \text { for any } v \in T_{x} X
$$

where $\||\cdot|\|,\||\cdot|\|_{0}$ are norms defined by $\ll, \gg, \ll, \gg_{0}$ respectively. This implies that $k_{x}$ is a compact operator.

[Q.E.D.]

Now we make clear the structure of $E^{-1}(0)$.

Proposition 2-3. Let $f$ be an isometry on $M$ with $f(N)=N$. Then the set of fixed points for $\left.f\right|_{N}$ is a disjoint union of totally geodesic submanifolds of $N$ each of which is a non-degenerate critical submanifold of $\Lambda_{N}(M, f)$ with index 0 . 
Proof. Here we must prove that each component $F_{i}(N, f)$ is nondegenerate and its index is 0 . (We write $F_{i}(N, f)$ simply by $F_{i}$ and we identify a point $p$ of $F_{i}$ with a constant map $\alpha \in \Lambda_{N}(M, f)$ such that $\alpha(t)$ $=p$ for all $t \in I$ in a natural way.)

We introduce the self-adjoint operator $h(E)_{\alpha}: T_{\alpha} \Lambda_{N}(M, f) \rightarrow T_{\alpha} \Lambda_{N}(M, f)$ at $\alpha \in \Lambda_{N}(M, f)$ defined by

$$
\ll h(E)_{\alpha} X_{\alpha}, Y_{\alpha} \gg=H(E)_{\alpha}\left(X_{\alpha}, Y_{\alpha}\right), \quad X_{\alpha}, Y_{\alpha} \in T_{\alpha} \Lambda_{N}(M, f) .
$$

Since $h(E)_{\alpha}$ is self-adjoint, we have $h(E)_{\alpha}\left(T_{\alpha} \Lambda_{N}(M, f)\right) \subset T_{\alpha} F_{i}^{\perp}$ for $\alpha \epsilon$ $F_{i}$ and so $h(E)_{\alpha}\left(T_{\alpha} F_{i}^{\perp}\right) \subset T_{\alpha} F_{i}^{\perp}$. Thus in order to see the non-degeneracy for $F_{i}$ we have only to show that restriction of $h(E)_{\alpha}$ on $T_{\alpha} F_{i}^{\perp}$ (denote it $\left.\bar{h}(E)_{\alpha}\right)$ is invertible.

At first we see that $\bar{h}(E)_{\alpha}: T_{\alpha} F_{i}^{\perp} \rightarrow T_{\alpha} F_{\alpha}^{\perp}$ is bijective. Note that the tangent space of $F_{i}$ at $\alpha \in F_{i}$ consists of constant vector fields $X_{\alpha} \in$ $T_{\alpha} \Lambda_{N}(M, f)$ along $\alpha$ and note that from (3) for any constant map $\alpha \in \Lambda_{N}(M, f)$

$$
H(E)_{\alpha}\left(X_{\alpha}, Y_{\alpha}\right)=\int_{0}^{1}\left\langle\nabla_{\alpha} X_{\alpha}(t), \nabla_{\alpha} Y_{\alpha}(t)\right\rangle d t, \quad X_{\alpha}, Y_{\alpha} \in T_{\alpha} \Lambda_{N}(M, f) .
$$

Then from (4) $\operatorname{ker} h(E)_{\alpha}=\left\{X_{\alpha} \in T_{\alpha} \Lambda_{N}(M, f) \mid \nabla_{\alpha} X_{\alpha}(t)=0\right.$ for almost everywhere for $\alpha \in F_{i}$. If $X_{\alpha} \in \operatorname{ker} h(E)_{\alpha}$, then $X_{\alpha}(t)$ is constant in $T_{\alpha(0)} N$ because $\nabla_{\alpha} X_{\alpha}(t)$ as a curve $T_{\alpha(0)} N$ at $\alpha \in F_{i}$ is just $X_{\alpha}^{\prime}(t)$. Thus we have $X_{\alpha} \in T_{\alpha} F_{i}$ because $f_{*}\left(X_{\alpha}(0)\right)=X_{\alpha}(1)$ and therefore $\operatorname{ker} h(E)_{\alpha} \subset T_{\alpha} F_{i}$. On the other hand, we have also $T_{\alpha} F_{i} \subset \operatorname{ker} h(E)_{\alpha}$ from (4) so that $\operatorname{ker} h(E)_{\alpha}=T_{\alpha} F_{i}$. Hence $h(E)_{\alpha}: T_{\alpha} F_{i}^{\perp} \rightarrow T_{\alpha} F_{i}^{\perp}$ is bijective.

Consequently $h(E)_{\alpha}$ is invertible because $h(E)_{\alpha}$ is actually a Fredholm operator by Lemma 2-2. Lastly since $H(E)_{\alpha}$ is semipositive definite for $\alpha \in F_{i}$, we have that the index of $F_{i}$ is 0 .

[Q.E.D.]

To get a very important property we need the following lemma.

Lemma 2-4 (Generalized Morse Lemma. W. Meyer [12]).

Let $\pi: X \rightarrow N$ be a differentiable Hilbert bundle over a compact connected manifold and let $\langle$,$\rangle be a Riemannian metric for this bundle. Let$ $K: X \rightarrow R$ be a differentiable function such that a zero section $\tilde{N}$ of $N$ is a non-degenerate critical submanifold for $K$ with level 0 . Then there exists a tubular neighborhood $\nu_{\varepsilon}=\{v \in X \mid\|v\|<\varepsilon\}$ for $N$ in $X$, a fibre preserving diffeomorphism $\Psi: \nu_{\varepsilon} \rightarrow \Psi\left(\nu_{\varepsilon}\right)$ and an orthogonal projection $P$ such that

$$
K \circ \Psi(v)=\|P v\|^{2}-\|(I-P) v\|^{2}, \quad \text { for any } v \in \nu_{s}
$$


where $\|\cdot\|$ is the norm defined by $\langle$,$\rangle .$

In our case consider a tubular neighborhood $\nu_{\varepsilon_{i}}$ for $F_{i}(N, f)$ and $K=$ $E \circ \exp \mid \nu_{\varepsilon_{i}}$ where exp is the exponential map defined by the Riemannian metric of $\Lambda_{N}(M, f)$ when $N$ is compact. Then by this lemma we have

$$
E \circ \Phi_{i}(v)=\left\|P_{i} v \mid\right\|^{2}-\left\|\left(I_{i}-P_{i}\right) v\right\|^{2}, \quad \text { for any } v \in \nu_{s_{i}}
$$

where $\Phi_{i}=\exp \circ \Psi_{i}: \nu_{\varepsilon_{i}} \rightarrow \Phi_{i}\left(\nu_{\varepsilon_{i}}\right) \subset \Lambda_{N}(M, f)$ is a diffeomorphism and $\|\mid \cdot\|$ is the norm defined by the Riemannian metric on $\Lambda_{N}(M, f)$.

Now put $\Phi=\bigcup_{i} \Phi_{i}$ and $\varepsilon=1 / 2 \min \varepsilon_{i}$, then we have $\Phi\left(\bigcup_{i} \bar{\nu}_{\varepsilon}^{i}\right)=E^{-1}[0, \varepsilon]$ because $E \circ \Phi_{i}(v)=\left\|P_{i} v \mid\right\|^{2}$ from index $F_{i}(N, f)=0$ as seen in Proposition 2-3.

By using Condition (C) we can find $\varepsilon>0$ such that $E^{-1}[0, \varepsilon]$ has no critical point except level 0 . And moreover since $\left\{0\right.$-section of $\left.\nu_{\varepsilon}^{i}\right\}$ is a strongly deformation retract of $\nu_{\varepsilon}^{i}$, we have

Proposition 2-5. There exists an $\varepsilon>0$ such that $F(N, f)$ is a strongly deformation retract of $E^{-1}[0, \varepsilon]$.

From the above proposition we state the following key lemma for the existence of critical points for $E$.

Lemma 2-6. Let $M$ be a complete Riemannian manifold, $N$ a connected compact closed submanifold of codimension 1 with $\partial N=\phi$ and $f$ an isometry of $M$ such that $f(N)=N$ and $F(N, f) \neq \phi$. If there exists no non-trivial geodesic with the same angle, then the inclusion map $i: F(N, f) \rightarrow \Lambda_{N}(M, f)$ is a homotopy equivalence. Moreover there exists a non-trivial geodesic with the same angle if $F(N, f)=\phi$.

Proof. This proof is quite similar to a part of Lemma 3-4 in ([2]). Since $F(N, f)$ and $\Lambda_{N}(M, f)$ are $A N R$ 's, we have only to show that the induced homomorphism $i_{q}: \pi_{q}(F(N, f)) \rightarrow \pi_{q}\left(\Lambda_{N}(M, f)\right)$ gives an isomorphism for all integer $q \geqq 0$.

Choose a base point in $F(N, f)$ and let that be a base point for $\Lambda_{N}(M, f)$. Let $S^{q}$ be a representative element of $\pi_{q}(F(N, f))$ such that $i_{q}\left(\left[S^{q}\right]\right)=\left[\tilde{S}^{q}\right]=0$ in $\Lambda_{N}(M, f)$. Since $\lim _{t \rightarrow \infty} \max _{\alpha \in \tilde{S} q} E\left(\eta_{t}(\alpha)\right)$ must be a critical level for $E$ by Condition (C), we have $\lim \max E\left(\eta_{t}(\alpha)\right)=0$ from the assumption of this lemma where $\eta_{t}$ is a flow defined by the vector field (-grad $\left.E\right)$. And so for large enough $t_{0} \in R_{+}$there exists an $\varepsilon>0$ such that $\eta_{t_{0}}\left(\tilde{S}^{q}\right) \subset E^{-1}[0, \varepsilon]$ and $E^{-1}[0, \varepsilon]$ is deformable to $F(N, f)$ by Proposition 2-4. Thus $S^{q}$ is null homotopic 
in $F(N, f)$ and so $i_{q}$ is a monomorphism. Conversely let $\tilde{S}^{q}$ be any representative element of $\pi_{q}\left(\Lambda_{N}(M, f)\right)$, then in the same way as above we can construct a representative element $S^{q}$ of $\pi_{q}(F(N, f))$ such that $i_{q}\left(\left[S^{q}\right]\right)=$ $\left[\tilde{S}^{q}\right]$. Hence $i_{q}$ is an epimorphism. Consequently $i_{q}$ is an isomorphism.

As to the last half Condition (C) implies that the energy function on $\Lambda_{N}(M, f)$ attains its infimum on any component of $\Lambda_{N}(M, f)$ (See [13]), and the inf points are of course critical points for $E$ which values of $E$ are different from 0 because $F(N, f)=\phi$. And so it is a non-trivial geodesic with the same angle.

[Q.E.D.]

Now we have

Theorem 2-7. Let $M$ be a complete Riemannian manifold, $N$ a connected compact closed submanifold of codimension 1 with $\partial N=\phi$ and $f$ an isometry on $M$ with $f(N)=N$. If one of the following conditions is satisfied, then there exists a non-trivial geodesic with the same angle for $N$. The conditions are

(1) $\sharp F(N, f)=0$

(2) $2 \leqq \# F(N, f)<\infty$ and $\pi_{1}(M)=\{0\}$

where \# is the number of elements of $F(N, f)$.

Proof. The case (1) is evident by the last half of Lemma 2-6. Since the fibration $\Lambda_{N}(M, f) \rightarrow G(M, N, f) \rightarrow N$ has a fibre $\Lambda_{p}(M)=\left\{\alpha \in \Lambda_{N}(M, f) \mid \alpha(0)\right.$ $=\alpha(1)=p\}$ where $f(p)=p$ is the base point in $N$, the homotopy exact sequence

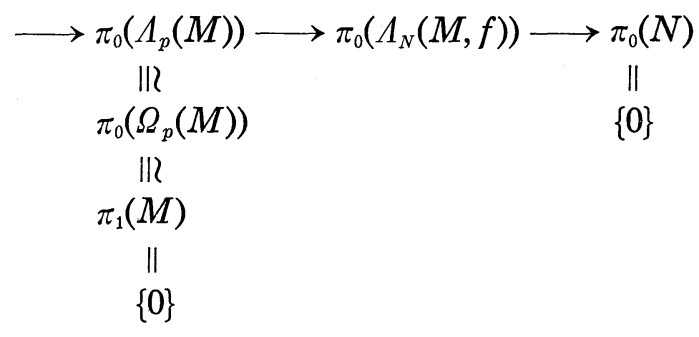

gives $\pi_{0}\left(\Lambda_{N}(M, f)\right)=0$ because $\Lambda_{p}(M)$ is the same homotopy type to the loop space $\Omega_{p}(M)$ at $p$. By the assumption $\pi_{0}(F(N, f)) \neq 0$ and so we have the conclusion by Lemma $2-6$.

[Q.E.D.]

Remark. When $\sharp F(N, f)=+\infty$, we have $\operatorname{dim} F_{i}(N, f) \geqq 1$ for a component $F_{i}(N, f)$ of $F(N, f)$. Since $F_{i}(N, f)$ is a totally geodesic submanifold of $N$ as seen in Proposition 2-3, any geodesic on $F_{i}(N, f)$ is an $f$-invariant 
geodesic on $N$. Of course, this geodesic is not necessarily a geodesic on $M$.

Now consider an orientation for $M$, then this orientation induces an orientation for $N$. When $M$ is orientable, we can apply our consideration of the previous paper ([10], [11]) to this case.

Let $f$ be an isometry of $M$ with $f(N)=N$, then we denote a trace of an induced homomorphism $\left(\left.f\right|_{N}\right)_{k}: H_{k}(N, Q) \rightarrow H_{k}(N, Q)$ of $k$-th homology group by $\lambda_{k}(N, f)$. Then we have the following property.

Corollary 2-8. Let $M, N$ and $f$ be the same as Theorem 2-7. Suppose that $M$ is a simply connected orientable manifold of odd dimension and $f$ is orientation preserving. Then there exists a non-trivial geodesic with the same angle or a nontrivial f-invariant geodesic on $N$ if $\lambda_{k}(N, f)$ is even for $k=1 / 2(\operatorname{dim} M-1)$. In particular it is well if $H_{k}(N, Q)=0$.

Proof. By the Poincaré duality, we have $L\left(\left.f\right|_{N}\right)=2 \sum_{1}^{k-1}(-1)^{i} \lambda_{i}(N, f)$ $+(-1)^{k} \lambda_{k}(N, f)+2$ where $L\left(\left.f\right|_{N}\right)$ is the Lefschetz number of $\left.f\right|_{N}: N \rightarrow N$. On the other hand it is known that $L\left(\left.f\right|_{N}\right)$ equals the Euler number $\chi(F(N, f))$ of $F(N, f)$ ([9]). Thus if $\lambda_{k}(N, f)=$ even, $L\left(\left.f\right|_{N}\right) \neq 1$ and so $\chi(F(N, f)) \neq 1$. When $\chi(F(N, f)) \geqq 2$, our conclusion follows from Theorem 2-7 and the above Remark. If $\chi(F(N, f))=0$, then there is no fixed points or $F(N, f)$ contains non-isolated fixed point set. Hence in the first case our conclusion follows from (1) of Theorem 2-7 and in the second case it follows from the above Remark.

[Q.E.D.]

Further when we consider an order of $\left.f\right|_{N}$ where it is defined by the minimal integer $n$ such that $\left.f^{n}\right|_{N}$ is homotopic to the identity, we can describe which isometries satisfy the condition " $\lambda_{k}(N, f)=$ even" of Corollary 2-8 in the same way as our previous paper ([11]). We denote the order of $\left.f\right|_{N}$ by $\operatorname{ord}(N, f)$.

(1) $\operatorname{rank} H_{k}(N, Z)=$ even, $\quad$ ord $(N, f)=2,4,8$

(2) $\operatorname{rank} H_{k}(N, Z)=2, \quad$ ord $(N, f) \neq 0(\bmod 3)$

(3) $\operatorname{rank} H_{k}(N, Z)=3, \quad \operatorname{ord}(N, f) \neq 0(\bmod 2)$

$$
f \text { has no eigenvalue } 1
$$

where $k>1$ and $Z$ is the group of integers.

In the case of $\operatorname{dim} M=$ even, the fixed point $F(N, f)$ is not isolated as seen in our paper ([10]). Hence there exists a non-trivial $f$-invariant geodesic (actually $f$-fixed geodesic) of $N$ if $F(N, f) \neq \phi$. 


\section{§3. Self-intersecting geodesic}

As seen in Remark of $\S 1$ a geodesic with the same angle is a selfintersecting geodesic if $F(N, f)=N$ and we call it a geodesic loop. Here we consider the existence of geodesic loops. And we give an answer for our problem in a sence.

Proposition 3-1. Let $M$ be a complete Riemannian manifold and let $N$ be a connected compact closed submanifold of codimension 1 with $\partial N=$ $\phi$. If $f$ is an isometry of $M$ with $F(N, f)=N$, then we have

$$
\pi_{n}\left(\Lambda_{N}(M, f)\right) \cong \pi_{n}(N)+\pi_{n+1}(M) \quad(n \geqq 2)
$$

and moreover

$$
0 \longrightarrow \pi_{2}(M) \longrightarrow \pi_{1}\left(\Lambda_{N}(M, f)\right) \longrightarrow \pi_{1}(N) \longrightarrow 0
$$

is exact.

Proof. It is known that the inclusion map $\Lambda_{N}(M, f) \longrightarrow C_{N}^{0}(M, f)$ is a homotopy equivalence where $C_{N}^{0}(M, f)=\left\{\alpha \in C^{0}(I, M) \mid \alpha(0) \in N, \alpha(1)=f(\alpha(0))\right\}$ is a Banach manifold of continuous maps with the uniform topology. And so we consider $C_{N}^{0}(M, f)$ instead of $\Lambda_{N}(M, f)$. We have an exact sequence for the fibration $P: C_{N}^{0}(M, f) \longrightarrow G(M, N, f)=\cdot \Delta(N)$ (diagonal), $P(\alpha)=$ $(\alpha(0), \alpha(1))=(x, x)$, with fibre as loop space $\Omega_{q}(M)$ where $q$ is the base point in $N$.

Consider now the commutative diagram,

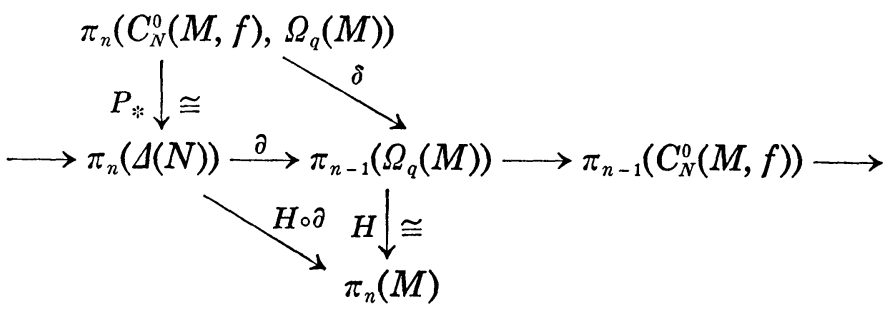

where $\delta$ is the boundary map in the exact sequence for the pair $\left(C_{N}^{0}(M, f)\right.$, $\left.\Omega_{q}(M)\right), H$ is the Hurewitz map and $P_{*}: \pi_{n}\left(C_{N}^{0}(M, f), \Omega_{q}(M)\right) \rightarrow \pi_{n}(U(N))$ is an isomorphism induced by projection $P$ for $n \geqq 1$. Now we see that $\partial$ is a zero homomorphism.

Let $E \in \pi_{n}\left(C_{N}^{0}(M, f), \Omega_{q}(M)\right)$ be represented by the map

$$
\xi:\left(I^{n}, \partial I^{n}, J^{n-1}\right) \longrightarrow\left(C_{N}^{0}(M, f), \Omega_{q}(M), q\right)
$$


where $I^{n}$ is the $n$-cube, $\partial I^{n}$ its boundary, and $J^{n-1}=I^{n-1} \times\{0\} \cup\left(\partial I^{n-1}\right)$ $\times I$. Now $\delta(\xi)=\partial \circ P_{*}(E)$ is represented by the restriction $\xi_{1}$ of $\xi$ to the bottom,

$$
\xi_{1}:\left(I^{n-1}, \partial I^{n-1}\right) \times\{1\} \longrightarrow\left(\Omega_{q}(M), q\right) .
$$

Evaluation of this map,

$$
\bar{\xi}_{1}:\left(I^{n-1} \times I, \partial I^{n}\right) \longrightarrow(M, q)
$$

represents $H \circ \delta(\Xi)=H \circ \partial \circ P_{*}(\Xi)$. Thus $H \circ \partial \circ P_{*}(\Xi)$ is represented by the restriction of the evaluation $\bar{\xi}$ of $\xi$ to $I^{n-1} \times I$

$$
\bar{\xi}:\left(I^{n}, \partial I^{n}, J^{n-1}\right) \times I \longrightarrow(M, M, q) .
$$

From this we see that $\bar{\xi}$ gives rise to a homotopy between $\xi$ restricted to $I^{n} \times\{0\} \cup I^{n-1} \times I \cup I^{n} \times\{1\}$ and the constant map.

By the way $\left.\bar{\xi}\right|_{I^{n} \times\{0\} \cup I^{n-1} \times I \cup I^{n} \times\{1\}}$ represents $-P_{1 *} \circ P_{*}(\Xi)+H \circ \partial \circ P_{*}(\Xi)$ $+P_{2 *} \circ P_{*}(\Xi)$ where $P_{i}: \Delta(N) \longrightarrow N$ is a $i$-th factor projection. Since $P_{1}$ $=P_{2}$, we have $H \circ \partial \circ P_{*}(\Xi)=0$ and this implies that $\partial$ is a zero homomorphism. Thus we have the following exact sequence,

$$
0 \longrightarrow \pi_{n}\left(\Omega_{q}(M)\right) \longrightarrow \pi_{n}\left(C_{N}^{0}(M, f)\right) \longrightarrow \pi_{n}(\Delta(N)) \longrightarrow 0
$$

for all $n \geqq 0$. Since we have a trivial cross-secton $s: \Delta(N) \rightarrow C_{N}^{0}(M, f)$ defined by $s(x, x)(t)=x$ for all $t \in I$, we have

$$
\pi_{n}\left(C_{N}^{0}(M, f)\right) \cong \pi_{n}\left(\Omega_{q}(M)\right)+\pi_{n}(\Delta(N)) \quad \text { for } n \geqq 2 .
$$

On the other hand, since

$$
\begin{aligned}
\pi_{n}\left(\Omega_{q}(M)\right) & \cong \pi_{n+1}(M) \\
\pi_{n}(\Delta(N)) & \cong \pi_{n}(N) \\
\pi_{n}\left(C_{N}^{0}(M, f)\right) & \cong \pi_{n}\left(\Lambda_{N}(M, f)\right),
\end{aligned}
$$

we have the conclusion of theorem.

[Q.E.D.]

TheOREM 3-2. Let $M$ be a complete Riemannian manifold and let $N$ be a connected compact closed submanifold of codimension 1 with $\partial N=\phi$. If $\pi_{k}(M) \neq 0$ for some $k \geqq 1$ and there exists an isometry $f$ with $F(N, f)=$ $N$, then there exists a non-trivial geodesic loop starting from $N$ with the same angle for $N$.

Proof. If there exists no non-trivial geodesic with the same angle, we have an isomorphism induced by the inclusion $i: F(N, f)=N \rightarrow C_{N}^{0}(M, f)$, 
$i_{*}: \pi_{n}(N) \cong \pi_{n}\left(C_{N}^{0}(M, f)\right)(n \geqq 0)$. And we have the commutative diagram,

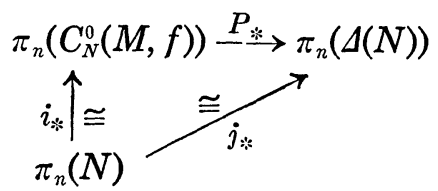

where $j_{*}$ is an isomorphism induced by the map $j: N \rightarrow \Delta(N)$ where $j(x)$ $=(x, x)$. Thus $P_{*}: \pi_{n}\left(C_{N}^{0}(M, f)\right) \rightarrow \pi_{n}(\Delta(N))$ is an isomorphism. On the other hand, since the sequence

$$
0 \longrightarrow \pi_{n}\left(\Omega_{q}(M)\right) \longrightarrow \pi_{n}\left(C_{N}^{0}(M, f)\right) \longrightarrow \pi_{n}(\Delta(N)) \longrightarrow 0
$$

is exact as seen in the proof of Proposition $3-1, \pi_{n}\left(\Omega_{q}(M)\right)=0$ for all $n \geqq$ 0 . Therefore we have $\pi_{n}(M)=0$ for all $n \geqq 1$ so that it contradicts the assumption of theorem.

[Q.E.D.]

For example let $M$ be a product manifold $N_{1} \times N_{2}$ with the product metric where $N_{1}$ is a connected compact manifold of (dim $\left.M-1\right)$ dimension. Then we can apply this theorem for the isometries id $\times g$ where $g$ is an arbitrary isometry of $N_{2}$.

Remark. In the case $f=$ identity we have Theorem B of Introduction. Moreover maybe we can show the existence of geodesic loops under more weak conditions and we shall do it elsewhere.

From this theorem we see the following corollaries.

Corollary 3-3. Assume that $M$ and $N$ are the same as Theorem 3-2 and there exists an isometry $f$ with $F(N, f)=N$. If $M$ is compact, then there exists a non-trivial geodesic loop starting from $N$ with the same angle for $N$.

Proof. Since $M$ is compact, $\pi_{n}(M) \neq 0$ for some $n \geqq 1$.

Corollary 3-4. Assume that $M$ and $N$ are the same as Theorem 3-2 and there exists an isometry $f$ with $F(N, f)=N$. If $M$ is the same homotopy type as $N$, then there exists a non-trivial geodesic loop starting from $N$ with the same angle for $N$.

Proof. If $M$ is the same homotopy type as $N, \pi_{n}(M) \cong \pi_{n}(N)$ for $n \geqq$ 0. Since $M$ is compact, $\pi_{n}(M) \cong \pi_{n}(N) \neq 0$ for some $n \geqq 1$. [Q.E.D.]

Here we have an answer for our proposed problem in a sence. 
Corollary 3-5. Let $M$ be a complete Riemannian manifold and let $N$ be a connected compact closed submanifold of codimension 1 with $\partial N=\phi$. Then there exists a non-trivial geodesic with the same angle.

Proof. If $M$ is the same homotopy type as $N$, then there exists a nontrivial geodesic (=geodesic loop) with the same angle by applying Corollary 3-4 for $f=$ identity. On the other hand if $M$ is not the same homotopy type as $N$, then it is known that there exists a geodesic which starts perpendicularly from $N$ and ends perpendicularly in $N$ by K. Grove ([4]) (The same results are shown by S. Kawai [6]). Thus we have our conclusion.

[Q.E.D.]

\section{§4. N-I-geodesic}

Here we consider a special case of geodesics with the same angle and so we give some conditions for the existence of such a geodesic.

Let $M$ be a complete Riemannian manifold, $N$ a closed submanifold and $f$ an isometry on $M$ with $f(N)=N$.

Definition 4-1. A geodesic $\alpha: I \rightarrow M$ satisfying the condition $\alpha(1)=$ $f(\alpha(0))$ and $(\alpha(0), \alpha(1)) \in N \times N$ is called a normal connected invariant geodesic (we say it " $N$ - $I$-geodesic of $f$ ") if it satisfies the following two conditions.

(1) $(\dot{\alpha}(0),-\dot{\alpha}(1)) \in T_{\alpha(0)} N^{\perp} \times T_{\alpha(1)} N^{\perp}$

(2) $\alpha$ is invariant under $f$.

For example let $S^{2}$ be a 2-dimensional standard sphere, $N=S^{2} \cap$ $\{(y, z)$-plane $\}$ and $f=180^{\circ}$-rotation with the fixed points $(0,0, \pm 1)$, then there exists a $N$-I-geodesic of $f$.

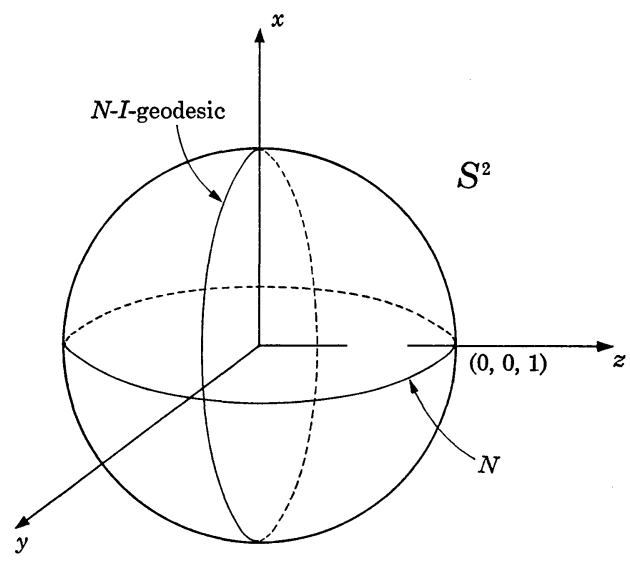


On the other hand let $T^{2}$ be a flat torus, $f$ be an $180^{\circ}$-rotation about its barycenter and $N=$ horizontal line, then there does not exists a $N-I$ geodesic of $f$.

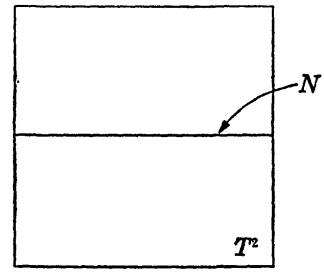

Thus in general there does not exists $N$-I-geodesic for any given $M$, $N$ and $f$.

Lemma 4-1. Let $M$ be a complete Riemannian manifold, $N$ a closed submanifold of codimension 1 with $\partial N=\phi$ and $f$ an isometry of $M$ with $f(N)=N$. If $\alpha \in \Lambda_{N}(M, f)$ is a critical point for $E$ with $\dot{\alpha}(0) \perp T N$, then $\alpha$ is a $N$-I-geodesic of $f$.

Proof. It follows by Theorem 1-3 that $\alpha$ is a geodesic with the same angle and $\dot{\alpha}(1) \perp T N$ when $\dot{\alpha}(0) \perp T N$. And so $f_{*} \dot{\alpha}(0)$ and $\dot{\alpha}(1)$ are normal to $T_{\alpha(1)} N$. Since the codimension of $N$ is 1 and $\|\dot{\alpha}(0)\|=\|\dot{\alpha}(1)\|$, we have $f_{*} \dot{\alpha}(0)= \pm \dot{\alpha}(1)$. This implies that $\alpha$ is invariant under $f$.

Let $\Gamma(M)$ be a group of isometry of $M$ and let $\Gamma_{N}(M)$ be a subgroup of $\Gamma(M)$ such that any element of $\Gamma_{N}(M)$ leaves $N$ invariant.

TheOREM 4-2. Let $M$ be a complete Riemannian manifold and let $N$ be a connected compact closed submanifold of codimension 1 with $\partial N=\phi$. If $\Gamma_{N}(M)$ acts on $N$ transitively and $N$ is not the same homotopy type as $M$, then there exists an element $f$ of $\Gamma_{N}(M)$ such that it has a N-I-geodesic.

Proof. It is known by K. Grove ([4]) that if $N$ is not the same homotopy types as $M$, then there exists a non-trivial geodesic $\alpha$ with $(\dot{\alpha}(0)$, $-\dot{\alpha}(1)) \in T_{\alpha(0)} N^{\perp} \times T_{\alpha(1)} N^{\perp}$. Since $\Gamma_{N}(M)$ is transitive, there exists an element $f \in \Gamma_{N}(M)$ with $f(\alpha(0))=\alpha(1)$. And moreover this $\alpha$ is a critical point for $E: \Lambda_{N}(M, f) \rightarrow R$ so that $\alpha$ is a $N$-I-geodesic by Lemma $4-1$. [Q.E.D.]

At once we can find easy examples;

(a) $M=S^{2}, \quad N=$ equator.

(b) $M=R^{3}, \quad N=S^{2}$.

Acknowledgment. The author wishes to thank Professor Y. Shikata 
and Professor M. Adachi for their encouragements, and Professor $\mathbf{M}$. Tanaka for his kindly advices.

\section{REFERENCES}

[1] Flaschel, P., und Klingenberg, W., Riemannsche Hilbertmannigfaltigkeiten. Periodische Geodätische, Lecture Notes in Math. Vol. 282, Springer (1972).

[2] Grove, K., Condition(C) for the energy integral on certain path-spaces and applications to the theory of geodesics, J. Diff. Geom., 8 (1973), 207-223.

[ 3 ] — - Isometry-invariant geodesics, Topology, 13 (1974), 281-292.

[4] — Geodesics satisfying general boundary conditions, Comment. Math. Helv., 48 (1973), 376-381.

[ 5 ] Karcher, H., Closed geodesics on compact Riemannian manifolds, Nonlinear functional analysis (J. T. Schwartz) Chapter VIII, Gordon and Breach, New York (1969).

[6] Kawai, S., Double normals of submanifolds in Riemannian manifolds. Master Thesis (Kyoto University) (1977).

[ 7 ] Klingenberg, W., Lectures on closed geodesics, Math. Inst. der Univ. Bonn (1976).

[ 8 ] Kobayashi, S., Fixed points of isometries, Nagoya Math. J., 13 (1958), 63-68.

[ 9 ] — Transformation groups in differential geometry, Ergebnisse der Math., Bd. 70 (1972).

[10] Kurogi, T., Riemannian manifolds admitting some geodesics, Proc. Japan Acad., 50 (1974), 124-126.

[11] — Riemannian manifolds admitting some geodesic II, ibid., 52 (1976), 7-9.

[12] Meyer, W., Kritsche Mannigfaltigkeiten in Hilbertmannigfaltigkeiten, Math. Ann., 190 (1967), 45-66.

[13] Palais, R. S., Morse theory on Hilbert manifolds, Topology, 2 (1963), 299-340.

Fukui University 